NASA/CR. $1998 \quad 207690$

1151-97046

$$
M N=34-C R
$$

AIAA 98-2326

Mixing Enhancement by Tabs in Round Supersonic Jets

\author{
John M. Seiner \\ NASA Langley Research Center \\ Hampton, VA USA \\ C.E. Grosch \\ Old Dominion University \\ Norfolk, VA USA
}

4'th AIAA/CEAS Aeroacoustics Conference

Toulouse, France

June 2-4, 1998 
$\vdots$ 


\title{
Mixing Enhancement by Tabs in Round Supersonic Jets
}

\author{
John M. Seiner* \\ Aeroacoustics Branch \\ NASA Langley Research Center \\ Hampton, Virginia, USA \\ and \\ C.E. Grosch \\ Department of Oceanography and Computer Science \\ Old Dominion University \\ Norfolk, VA, USA
}

\begin{abstract}
The objective of this study was to analyze jet plume mass flow entrainment rates associated with the introduction of counter-rotating streamwise vorticity by prism shaped devices (tabs) located at the lip of a nozzle. We have examined the resulting mixing process through coordinated experimental tests and numerical simulations of the supersonic flow from a model axisymmetric nozzle. In the numerical simulations, the total induced vorticity was held constant while varying the distribution of counter-rotating vorticity around the nozzle lip trailing edge. In the experiment, the number of tabs applied was varied while holding the total projected area constant. Evaluations were also conducted on initial vortex strength. The results of this work show that the initial growth rate of the jet shear layer is increasingly enhanced as more tabs are added, but that the lowest tab count results in the largest entrained mass flow. The numerical simulations confirm these results.
\end{abstract}

\section{Introduction}

Mixing enhancement of high and low speed streams is utilized as a means to improve efficiency of supersonic combustors, reduce aircraft signatures. and control high speed jet noise. Although the present program's primary goal was to attain jet signature reduction through enhanced plume mixing rates, the primary focus of this paper and our laboratory's research is the development of performance efficient jet noise suppression technology.

Various methods to achieve improved mixing have been studied for the applications mentioned above.

* Associate Fellow, AIAA

Copyright Notice: This paper is declared a work of the United States Government and is not subject to copyright protection in the United States
One common method has been the introduction of streamwise vorticity by prism shaped wedges. Such devices have been successfully investigated experimentally by Dolling, Fournier, and Shau ' ${ }^{\prime}$ and numerically by Drummond, Carpenter, Riggins, and Adams $^{2}$ for application to the hydrogen fueled supersonically combusting ramjet.

Ahuja and Brown ${ }^{3}$ investigated how these devices, when located on the jet nozzle lip, could be utilized to enhance mixing of high-speed jet flow with the surrounding medium. They named their device a tab, after those commonly used to achieve screech control in supersonic jets. They inferred a large increase in jet flow entrainment for both heated and unheated jets through observation of enhanced decay of jet centerline velocity. The near field jet spectra indicated, that in addition to screech control, low frequency jet noise was also reduced. Samimy, Zaman, and Reeder ${ }^{4}$ and Zaman, Reeder, and Samimy ${ }^{5}$ further investigated the properties of enhanced mixing using tabs. In these studies they measured the streamwise mass flux and found tab configurations capable of entraining 1.5 times the mass flux of the baseline axisymmetric nozzle. The design of their tab geometry was based on the production of large-scale streamwise vorticity, which promotes mixing due to an increase in perimeter contact between low and high-speed streams. Their tab geometry was not designed to excite naturally occurring jet shear layer instabilities. In both these studies the enhanced jet mixing rates were used as a means for reducing jet noise, although these studies have not obtained the required acoustic confirmation.

At the outset of this study it was clear that no theoretical or semi-empirical method existed that could relate tab design to enhanced mixing rate and noise reduction. Enhanced mixing has not always led to noise reduction in the appropriate metric ${ }^{6}$. Enhanced mixing was found to be an acceptable method when the far field noise is dominated by eddy Mach wave emission. When 
considering initial tab designs it was uncertain how to select the number or spacing of these devices, penetration depth into the initial jet shear layer, and ramp angle of the tabs. Zaman, Reeder, and Samimy ${ }^{4}$ had determined that those tabs that were inclined $45^{\circ}$ downstream of the nozzle exit produced the largest mass flow entrainment rates.

In the present study we have examined the mixing process associated with tab-like devices located on the jet nozzle lip through coordinated experimental and numerical simulations. Because of program constraints, the nozzle geometry chosen was that of the J-85 engine nozzle. Tabs were designed with $45^{\circ}$ ramp angles and penetration depths that produced $3 \%$ projected area blockage at the nozzle exit with 2, 4 and 6 tabs mounted on the nozzle lip trailing edge. This permitted examination of the distribution of total induced counter-rotating vorticity on mixing enhancement. To examine the effect of total induced vorticity strength; one set of tabs was designed with $8 \%$ projected area using 2 tabs. The fence tabs of Ahuja and Brown ${ }^{3}, 3.57 \%$ projected area, were also applied. These tabs had ramp angles of $90^{\circ}$. Experimental measurements were acquired for all these configurations to determine mass flow entrainment. Numerical simulations were performed using a Navier-Stokes code for the 2 and 4 tab configurations.

The results of this work show that the initial growth rate of the jet shear layer is increasingly enhanced as more tabs are added, but that the lowest tab count results in the largest entrained mass flow. The extent to which the numerical simulations confirm these results is detailed in this paper.

\section{Experimental Description}

The experimental research was conducted in the NASA Langley Jet Noise Laboratory's (JNL) Small Anechoic Jet Facility (SAJF). This facility is used to study small scale nozzles up to $5.08 \mathrm{~cm}$. The facility has an anechoic test section and capability to electrically heat air for nozzle research to $811^{\circ} \mathrm{K}$. An indraft co-flow nozzle $(60.96 \mathrm{~cm}$ diameter) powered by an exhaust fan produces a maximum co-flow velocity of $6 \mathrm{~m} / \mathrm{sec}$.

For the tests reported in this paper, an axisymmetric round nozzle was constructed with exit diameter of $3.259 \mathrm{~cm}$. The convergent internal nozzle geometry of the axisymmetric nozzle was similar to the J-85 engine nozzle in the cruise power setting. The nozzle was operated in still air at nozzle pressure ratio's to produce fully expanded Mach numbers, $M_{j}$, of $0.37,0.85$, and 1.3 . Only the results at $\mathrm{M}_{\mathrm{j}}=1.3$ are reported in this paper. The flow was unheated. In addition to the baseline round nozzle, additional nozzles were fabricated that had 2, 4, and 6 tabs located at the nozzle exit. These tabs were fabricated by wire EDM. Their geometry was prism shaped with downstream ramp angles of $45^{\circ}$ inclined into the initial jet shear layer. The projected area into the flow of each nozzle's total tab set was $3 \%$ of the nozzle's baseline area. In addition, a 2- tabbed nozzle was designed that had a blockage area equal to $8 \%$. Fence-like tabs with $3.57 \%$ blockage area, were applied to the baseline nozzle. The fence-like tabs were identical to those used by Ahuja and Brown ${ }^{3}$. Figure 1 shows a photograph of the baseline nozzle and those with 4 and 6 tabs with $3 \%$ total projected area blockage.

For these experiments a single supersonic total pressure probe was traversed in several downstream nozzle cross-plane locations to measure the total mass flux. These axial station locations corresponded to $1,3,5,8,12$, and 16 jet diameters. Since the jet airflow was unheated total temperature measurements were not required to measure mass flux. One deficiency of these experiments is that plume static pressure was not measured. However, the error is expected to be small due to the low jet Mach number. Beyond 5 jet diameters the plume static pressure is nearly equal to ambient test cell pressure. The ambient test cell pressure and charging station total temperature were used throughout along with the total pressure measurements to compute measured mass flow. A commercial turbine meter was used to provide actual nozzle mass flow being delivered to the nozzle charging station.

\section{Numerical Simulation Description}

The simulations used the compressible three-dimensional, time dependent

mass conservation equation

$$
\frac{\partial \rho}{\partial t}+\frac{\partial}{\partial x_{j}}\left(\rho u_{j}\right)=0
$$

the compressible three-dimensional, time dependent Navier-Stokes equations

$\frac{\partial\left(\rho u_{j}\right)}{\partial t}+\frac{\partial}{\partial x_{k}}\left(\rho u_{j} u_{k}\right)+\frac{\partial p}{\partial x_{j}}=\frac{\partial \sigma_{j k}}{\partial x_{k}}$,

with

$$
\sigma_{i j}=2 \mu\left(S_{j k}-\frac{1}{3} \delta_{j k} S_{l l}\right)
$$




$$
S_{j k}=\frac{1}{2}\left(\frac{\partial u_{j}}{\partial x_{k}}+\frac{\partial u_{k}}{\partial x_{j}}\right),
$$

and the three-dimensional, time dependent energy equation

$$
\begin{aligned}
& \frac{\partial}{\partial t}\left[\rho\left(e+\frac{1}{2} u_{j} u_{j}\right)\right] \\
& +\frac{\partial}{\partial x_{k}}\left[\rho u_{k}\left(e+\frac{1}{2} u_{j} u_{j}+\frac{p}{\rho}\right)\right] \\
& =\frac{\partial}{\partial x_{k}}\left(u_{j} \sigma_{j k}\right)+\frac{\partial}{\partial x_{k}}\left(\kappa \frac{\partial T}{\partial x_{k}}\right),
\end{aligned}
$$

together with the equation of state,

$$
p=\rho R T
$$

As usual, $\rho$ is the density, the $\left(u_{i}\right)$ are the velocity components, $p$ is the pressure, $R$ is the gas constant, $e=C_{V} T$ is the internal energy per unit mass, $T$ is the temperature, $\kappa$ is the thermal conductivity, $C_{V}$ is the specific heat at constant volume and $\mu$ is the viscosity coefficient. The viscosity is computed using Sutherland's law

$$
\mu=\mu_{O} T^{3 / 2} /((110.0+T)
$$

It is assumed that the bulk viscosity coefficient is zero and that $C_{V}$ and $C_{P}$, the specific heat at constant pressure, are constant. Finally, the Prandtl number is assumed constant and equal to 0.72 .

The computational configuration is a jet with a circular cross-section of diameter $\mathrm{D}=3.259$ $\mathrm{cm}$. exiting into a square duct of height and width H. Figure 2 is a schematic of the channel and coordinate system. The length of the channel was set to $20.00 \mathrm{D}$. The jet speed is $\mathrm{U}_{1}$ with temperature $T_{1}$. In these calculations $T_{1}=313.2^{\circ} \mathrm{K}$ $\left(104.0^{\circ} \mathrm{F}\right)$ and $U_{1}=461.14 \mathrm{~m} / \mathrm{s}$, yielding the Mach number of the jet $M_{1}=1.300$. The co-flow had a speed of $U_{2}$ and temperature $T_{2}=295.4^{\circ} \mathrm{K}\left(72.0^{\circ} \mathrm{F}\right)$. We could not set $\mathrm{U}_{2}=0$ because the disparity between the advective and sound speed in the coflow caused numerical instabilities. Instead we set $\mathrm{U}_{2}=25.0 \mathrm{~m} / \mathrm{s}$ yielding a co-flow Mach number $\mathrm{M}_{2}$ $=0.073$. With this Mach number there were no numerical instabilities. Some of the calculations were repeated with a somewhat higher, but still small, value of $\mathrm{M}_{2}$ and we observed only very minor differences in the results. All of the results reported here had $M_{2}=0.073$. With these conditions the convective Mach number

$$
M_{C}=\frac{U_{1}-U_{2}}{c_{1}+c_{2}} \text {, }
$$

was 0.624 . Finally the jet Reynolds number is

$$
\operatorname{Re}=\frac{\left(U_{1}-U_{2}\right)}{\left(\mu_{1} \rho_{1}\right)}
$$

With these conditions $\operatorname{Re}=8.39 \times 10^{5}$.

There was no turbulence model used in these calculations. Thus we assume the flow to be laminar. In the experiments of Samimy et. al. ${ }^{4}$ and Zaman et. al. ${ }^{5}$ some of the configurations were, in their words, "nominally laminar" and others "nominally turbulent". In both cases they found that the experimental results were virtually identical. It appears that the dominant physical mechanism is the presence of the strong streamwise vortices generated by the tabs. Thus we expect that the laminar flow modeling in our calculation will not change the overall structure of the results.

The equations are approximated by finite differences on a grid with $\left(\mathrm{N}_{\mathrm{x}}, \mathrm{N}_{\mathrm{y}}, \mathrm{N}_{\mathrm{z}}\right)$ points in the $(x, y, z)$ directions, respectively. The finite difference equations are solved by a time accurate MacCormack predictor-corrector scheme (Peyret and Taylor ${ }^{7}$ ).

\section{Modeling the Tabs}

Possible physical mechanisms by which tabs produce streamwise vortices have been discussed by Samimy, Zaman and Reeder ${ }^{4}$ and by Zaman, Reeder and Samimy ${ }^{5}$. One possible mechanism is the stripping and rolling up of the boundary layer by the tab. Another possible mechanism is the effect of an upstream pressure gradient caused by the presence of the tab. Either mechanism could produce streamwise vortices. For either mechanism, the direction of rotation of the vortex pair could be controlled by the orientation of the tabs ${ }^{4}$. Based on their experimental observations, Samimy et. al. ${ }^{4}$ conjectured that a delta or triangular shaped tab placed on edge of a jet with the apex leaning downstream would produce a pair of vortices of the "trailing vortex" type and if the apex were pointing upstream the pair of vortices would be of the "necklace vortex" type. In their terminology, the difference between these types is the sense of rotation. The sense of rotation of the 
"necklace vortex" pair is such that, between the vortex pair fluid is moved from the jet into the coflow while for the "trailing vortex" type, between the vortex pair fluid is moved from the co-flow into the jet. In a later study, Zaman et. al. ${ }^{5}$ were able to show, via flow visualization that tabs produced different flows depending on whether the apex of the delta tab pointed upstream or downstream. By way of comparison we note that Grinstein, et. al. ${ }^{8}$ use the term "delta-wing vortex" instead of "trailing vortex" and "mushroom vortex" for "necklace vortex". In this paper we adopt the terminology of Samimy et. al. ${ }^{4}$ and denote the trailing vortices as either "necklace" or "trailing".

In these calculations it was not possible to directly add small physical tabs to the geometry because of the limitations on the spatial resolution in the code. Instead we model their effect by assuming that each tab generates a pair of counter rotating vortices. We introduce a simple model flow to represent these vortices on the inflow boundary. Figure 3 is a sketch illustrating the basic geometry. The point $\left(x_{0}, y_{0}\right)$ is the center of one of the vortices. The point $\left(x_{j}, y_{k}\right)$ is any grid point on the inflow boundary. The radial distance between these points is $r$. We assume that the vortex at $\left(\mathrm{x}_{0}, \mathrm{y}_{0}\right)$ generates a circumferential velocity, $V_{\theta}$, at $\left(x_{j}, y_{k}\right)$ which is given by

$$
V_{\theta}=V_{0}\left(\frac{b}{r}\right)\left[1-e^{-(y / b)^{2}}\right] \text {, }
$$

with $b$ the scale of the vortex and $V_{0}$ the amplitude. Then at $\left(x_{j}, y_{k}\right)$ the cartesian components of the velocity are

$$
\begin{aligned}
& U_{j, k}=V_{\theta} \cos \theta \\
& V_{j, k}=V_{\theta} \sin \theta
\end{aligned}
$$

In a counter rotating vortex pair, one has a positive $V_{0}$ and the other has a negative $V_{0}$. At each grid point on the inflow boundary we sum up the contributions to $\left(U_{j, k}, V_{j, k}\right)$ from each of the vortices, yielding the total inflow values of $\left(U_{j, k}\right.$, $\mathrm{V}_{\mathrm{j}, \mathrm{k}}$ ). In a similar way we modify the inflow pressure field to account for the vortices. The sign of $V_{0}$ sets the sense of rotation of the vortex pair. If $V_{0}$ is positive the vortex pair is a "necklace" vortex pair and is a "trailing" vortex if $\mathrm{V}_{0}$ is negative. In all of these calculations $V_{0}$ was negative and the vortices were of the "trailing" type i.e. we are modeling tabs with the apex leaning downstream.

\section{Boundary and Initial Conditions}

In all cases the top, bottom and side-walls of the computational domain were taken to be impermeable, slip and insulated boundaries, eliminating wall boundary layers which are irrelevant to the jet dynamics. The inflow, outflow and wall boundary conditions are discussed in detail in Grosch, et. al. ${ }^{9}$ where results of grid refinement studies and tests of the inflow and outflow condition were presented. There we showed that these conditions do not effect our results in any significant way.

In this calculation the top, bottom and side-walls are computational boundaries and do not represent physical boundaries. Therefore we want to minimize the effect of these computational boundaries. To access the effect of the placement of these boundaries we carried out a series of tests in which the jet flow without tabs was simulated with the computational boundaries at different distances from the centerline of the jet. The jet has a diameter $\mathrm{D}=3.259 \mathrm{~cm}$. at inflow. In test $\mathrm{A}$ the width and height of the computational domain, $\mathrm{H}$ (see Figure 2) were set to $0.015 \mathrm{~m}$ giving $\mathrm{H} / \mathrm{D}=$ 4.60. For the second and third tests $H$ was reduced thus moving the computational boundaries closer to the jet; in $\mathrm{B}, \mathrm{H}=0.0125 \mathrm{~m}$ giving $\mathrm{H} / \mathrm{D}=3.83$ and in $\mathrm{C}, \mathrm{H}=0.010 \mathrm{~m}$ giving $\mathrm{H} / \mathrm{D}=3.07$. In all of these tests the grid size in the $(x, y)$ plane was held constant so that the only change was the position of the computational boundaries. Some of the results are shown in Figure 4. The top panel shows, to scale, the jet at inflow and the positions of the computational boundaries for cases A, B and C. The dashed square in this panel delineates a region $0.05 \mathrm{~m}$ by $0.05 \mathrm{~m}$ centered on the jet axis. The two panels below show contours of the streamwise component of the velocity within the dashed square at 8 and 16 diameters downstream from the inflow boundary. Here $\left(x_{0}, y_{0}\right)$ is the position of the centerline of the jet. Solid, short dash, and long dash lines are used for the different cases. At $z$ / D $=8$ there are very minor differences between the results for cases A and B and small, but significant, differences between these and case $C$. At $\mathrm{z} / \mathrm{D}=$ 16 the results for cases $B$ and $C$ are markedly different from those of case $\mathrm{A}$. In cases $\mathrm{B}$ and $\mathrm{C}$ not only is there substantial distortion of the jet, but the jet has drifted slightly off the centerline. We concluded that in cases B and $C$ the computational boundaries were too close to the jet. Therefore all of the subsequent calculations were run with $\mathrm{H}=$ $0.150 \mathrm{~m}$, i.e. $\mathrm{H} / \mathrm{D}=4.60$. The plots we show below for the cases of 2 and 4 tabs will only show a region $\pm 1.50 \mathrm{D}$ centered on the jet axis, but it should be remembered that the computational 
boundaries are at $\pm 2.30 \mathrm{D}$ from the centerline. In all of our subsequent calculations we set $N_{x}=N_{y}$ $=101$ and $\mathrm{N}_{2}=201$.

\section{Experimental Axial Velocity Contours}

The measured jet exit Mach number profile of the baseline round nozzle is shown in Figure 5. The data shows that the initial jet boundary layer thickness is of the order of $4 \%$ of the jet exit diameter. This relatively thick boundary layer is attributed to the internal nozzle geometry design of the J-85 nozzle. The data of Figure 5 shows that the central flow from the nozzle is extremely uniform. All tab configurations have penetrations that extend well beyond the boundary layer. Specifically, nozzles with $3 \%$ projected area tabs all have a penetration depth equal to $11.1 \%$ of the jet exit diameter. The projected area for these nozzles is maintained constant by adjusting the base width of the tab at the nozzle lip. The nozzle with 2 -tabs and $8 \%$ projected area had a penetration depth equal to $26.8 \%$ of the jet exit diameter.

Figures $6 \mathrm{a}, 6 \mathrm{~b}, 6 \mathrm{c}$, and $6 \mathrm{~d}$ show the measured axial velocity contours obtained with the baseline nozzle at $z / D=1 ., 3 ., 8$, and 16 respectively. The nozzle is shown in scaled size on each of the figures. Between 361 and 441 probe locations were utilized to acquire this data in each cross plane. The measurement grid was uniform in both $\mathrm{x}$ and $\mathrm{y}$ directions, but had grid centers that increased in downstream locations to enable capture of the spreading turbulent jet. In general the jet aerodynamic centerline appears centered on the nozzle geometric centerline. By $z / D=16$ the jet flow appears to be slightly non-symmetric.

In a similar fashion, Figures $7 \mathrm{a}, 7 \mathrm{~b}, 7 \mathrm{c}$, and $7 \mathrm{~d}$ show the measured axial velocity contours obtained with the nozzle containing 4-tabs with $3 \%$ blockage at $z / D=1 ., 3 ., 8$, and 16 respectively. The figures are scaled to provide reference to the baseline nozzle. The tab location is shown on these figures in the orientation used throughout testing. As is evident from this data, the tabs introduce a well-defined perturbation of the jet flow. Planar imaging of this flow clearly showed the presence of counter rotating vorticity. It can be seen that as the flow evolves downstream, the jet becomes more elliptic, with a major axis that exists through the region with the largest tab separation. This will be better explained after viewing the numerical simulations. Figures $8 \mathrm{a}, 8 \mathrm{~b}, 8 \mathrm{c}$, and $8 \mathrm{~d}$ summarize the results obtained for the nozzle containing 6 -tabs with $3 \%$ blockage. In contrast to the 4 -tah nozzle, the flow is axisymmetric by $z / D=16$.

Figures 9 through 11 show the axial velocity contours for nozzles with 2 -tabs at $z, D=8$. and 16. Figure 9 shows results for those tabs with $3 \%$ projected area, while Figure 10 for $8 \%$. The Ahuja and Brown fence tabs results are shown in Figure 11. As can be seen, two strong vortex regions form independent of tab shape or size. The data also shows that each plume becomes nearly rectangular or oval with major axis that is rotated from the original plane of induced vorticity. It is also evident from these results that the highest velocity in any cross plane occurs off the centerline axis.

\section{Results of the Numerical Simulations}

Simulations were run with 2 and 4 tabs. The positions of the tabs are shown in Figure $12 ; 2$ tabs in panel, a, and 4 tabs in panel, b. In both of these panels the inflow region of the jet within the computational domain is also shown with the grid within the jet. The grid outside the jet is not shown for the sake of clarity; the resolution is the same as inside the jet. The positions of the tabs are shown by the black diamonds, which are of course, not to scale but are used only to denote the tab positions, which are the same as in the experiment. In panel, b, the upper and lower pair of tabs subtends an angle of $30^{\circ}$ with the side angles being $120^{\circ}$.

As discussed above, a pair of counter rotating vortices models the flow induced by each tab. The vortex parameters are (see equation 10) $\mathrm{V}_{0}$ and $b$, the vortex core radius. So far we have no experimental measurements of these quantities. Therefore we choose values which gave "reasonable" values of the maximum cross-plane velocity vectors and size of the vortices on $\mathrm{z} / \mathrm{D}=$ 1. These values are $V_{0}=160.0 \mathrm{~m} / \mathrm{s}$ and $\mathrm{b}=$ $0.0020 \mathrm{~m}$. At $\mathrm{z} / \mathrm{D}=1$ these gave a maximum of the cross-plane velocity vectors about of $45 \mathrm{~m} / \mathrm{s}$ (the jet speed in the core is $461 \mathrm{~m} / \mathrm{s}$ ) and a vortex diameter of approximately $0.20 \mathrm{D}$. We also varied these values to check the sensitivity of our results to them; it will be seen below that the results are not very sensitive to the values of $V_{0}$ and $b$.

The qualitative behavior of the flow in all cases was very similar. From the inflow boundary to $z / D=5$ or 6 the vortices were quite stable and the entire flow was almost steady. Beyond this location the vortices began to show an unstable, time varying behavior. This is precisely what we had seen in our previous simulations ${ }^{8}$. The pairs of counter rotating vortices interact with each other and evolve to an unsteady state. This is not a numerical instability. The calculation does not "blow up", the unsteady evolution just continues. Typically it takes about 4,000 time steps (about 6 $\mathrm{ms}$ ) for the calculated flow to reach an equilibrium 
state but we have run one case for 24,000 time steps without there being any "blow up".

An time averaged picture of the flow with 2 tabs is shown in Figures 13,14 , and 15 for $\mathrm{z} / \mathrm{D}=$ 3 ., 8., and 16 respectively. We averaged the velocity field on these planes over 1,000 time steps; this was about $1.50 \mathrm{~ms}$. There are two panels in each of the figures. The upper one showing contours of the streamwise component of the velocity and the lower the cross-plane velocity vectors. The maximum values of the magnitude of the cross-plane velocity vectors for these cases as well as all of the other cases are given in Table 1 . The pinching of the jet by the tab induced vorticity is clear in Figure $13(\mathrm{z} / \mathrm{D}=3)$ as is the slight distortion of the vortex pairs. It is observed that the counter-rotating pair of vortices induces ambient flow into the jet plume at the narrow separation of the tabs, and ejects fluid from the plume in the region of the largest tab separation. This pumping action of fluid gives rise to the basic downstream average plume shape. At $z / D=8$, Figure 14 , the jet has become two lobed. This is qualitatively what is seen in the experimental results (Figures 9,10, and 11) at the same streamwise location. However the computational jet is not spreading as fast as observed in the experiments. At this location the instability of the vortices is very apparent. At $\mathrm{z} / \mathrm{D}=$ 16 , Figure 15 , the instability has distorted the jet strongly and the vortices are very different from the structure at inflow. At this location the jet does not break up as the calculation proceeds. It remains a coherent structure but its shape oscillates with time. It appears that the jet is large enough that the flow may be being influenced by the computational boundaries at this location and further downstream.

Similar results for the flow with 4 tabs are shown in Figures 16,17 , and 18 for $z / D=3$., 8., and 16 respectively. The 4 tabs generate a different flow field from that with 2 tabs. At $\mathrm{z} / \mathrm{D}=3$, Figure 16, there is a finger of the jet at the top and bottom. The jet has almost uniformly spread sideways. This is very similar to the experimental results for 4 tabs at $z / D=3$ shown in Figure $7 b$. However, as for the 2-tab case, the spreading in the computational flow is not as great as in the experiment. The instability in the vortices is apparent at this location. By z / D $=8$, Figure 17, the jet has almost split into three and the vortices are seen to have been strongly disturbed. The experiments, Figure $7 \mathrm{c}$, show the central jet but not the side lobes; again there is more spreading in the experiments than in the computational results. Finally, at $z / D=16$, Figure 18 , the jet has lost most of its structure as has the vortices.
Mass Flow Entrainment Analysis

Figure 19 shows mean centerline velocities obtained with the baseline nozzle and those containing 2 tabs. Figure 20 shows the plume centerline behavior for all nozzles with tabs having $3 \%$ projected area. The centerline decay results would clearly mislead one if they are used to judge jet noise reduction, since the contour data of the previous figures show that peak centerline velocity occur off-axis. A more effective measure enhanced mixing for noise reduction is to integrate the measured axial velocity contours for mass flux through an axial station.

The results of this integration are shown in figures $21 \mathrm{a}$ and $21 \mathrm{~b}$. Figure $21 \mathrm{a}$ refers to nozzles with 2-tabs, and Figure $21 \mathrm{~b}$ nozzles with $3 \%$ projected area tabs. In both figures, the integrated mass flux at a given axial cross plane is normalized by the mass flux determined by measurement of nozzle mass flow from the turbine meter. The mass entrainment values in these figures are consistent with previous results of Ahuja and Brown, Samimy, et. al., and Zaman, et. al. We see that initially that each tabbed nozzle entrains more mass than the reference baseline nozzle. Those nozzles with two tabs entrain more low speed co-flow than those with a larger number of tabs. The entrainment is greatest for that configuration designed to produce the strongest counter-rotating vorticity. However, Figure $21 \mathrm{~b}$ shows that the nozzle with 6 tabs, while initially entraining more fluid than the baseline, eventually disrupts the normal process of turbulent mixing entraining less fluid than the baseline after $\mathrm{z} / \mathrm{D}=\mathbf{8}$.

In a similar fashion, Figure 22 shows the rate of increase in mass entrainment relative to supplied to the nozzle exit associated with the numerical simulations. Since the code contains no turbulence model, the baseline nozzle should entrain little of the co-flow since the jet shear layer is laminar. The results of Figure 22 confirm this, showing that the baseline nozzle mass flow rate increases slowly with downstream distance. In the numerical simulations, $W$, is the mass flux obtained by numerically integrating across the computational domain and $W_{0}$ is the mass flux in the jet at the inflow boundary. This is the same as in the experiments. The shear layer growth with counterrotating vorticity increases at a rate slightly less than that observed in the experiments, but it is found to depend on initial vortex strength as in the experiments. . For example, at $z / D=16, W / W_{0}$ is in the range of 3.0 to 3.5 from the experiments but about 2.5 from the calculations; i.e. the calculated mass flux is $20 \%$ to $40 \%$ lower than that measured. It may be that we should use a larger value of $V_{0}$. 
The Case $4 \mathrm{~b}$ is of interest since it shows that the mass entrainment can eventually decrease as was observed in the experiments with the 6-tab which induced the lowest strength vorticity into the shear layer.

To enable observation of the similarity between the numerical simulations and experimental measurements, the data of Figures 21 and 22 can instead be normalized by the measured mass flow of the baseline nozzle at the respective axial station. This to first order suppresses the influence of mixing by turbulent shear layer processes. The normalized mass flux increases monotonically with down stream distance. Figure 23 provides a summary of the experimental results where the mass flux in a given plane is normalized by the mass flux in that same plane associated with the baseline nozzle. As can be seen, those nozzles that have better distributed induced counter-rotating vorticity (i.e. more tabs), entrain more co-flow in the first 5 jet diameters than do those with a lower order. The total induced vorticity is approximately equal between 2 and 6 tabs with $3 \%$ projected area. As the turbulent jet shear layer grows, however, the relatively weaker vorticity associated with the higher tab count, actually leads to the development of a jet that entrains less co-flow than the baseline nozzle. Those nozzles with 2 -tabs initially entrain less than the baseline, but downstream of the jet potential core appear to have permanently altered the mass flow entrainment rate of the baseline nozzle.

Figures 24a and $24 \mathrm{~b}$ show how the numerical simulations compare to experimental observation. Figure 24a shows good comparison between experiment and the simulations for nozzles with 2-tabs. In the simulation the maximum crossstream velocity was $46.06 \mathrm{~m} / \mathrm{sec}$. at $\mathrm{z} / \mathrm{D}=1$. This is produced from selection of $V_{0}=160.0$ and $b=$ 0.002 . The computed value for $W / W_{B A S E}$ at $z / D=$ 16. Is high most likely due to influence of the computational boundary. The case labeled 4(a) in Figure $24 \mathrm{~b}$ also had $\mathrm{V}_{0}=160.0$ and $\mathrm{b}=0.002$; while case $4(\mathrm{~b})$ had $\mathrm{V}_{0}=120.0$ and $\mathrm{b}=0.002$; and case $4(c)$ had $V_{0}=160.0$ and $b=0.004$. It is clear that the mass flux is not very sensitive to selection of the tab model parameters. The initial growth of the shear layer is numerically reproduced in the jet potential core, but beyond over predicts the growth due to vorticity.

\section{Nozzle Discharge Coefficients}

We have shown how induced counterrotating vorticity can be used to enhance jet mass flow entrainment rates. Since the JNL SAJF does not have a balance, the present experiments cannot address thrust loss. However, it is possible to evaluate the change in nozzle discharge coefficient. Table II shows a comparison of the measured nozzle discharge coefficient to relative to that of the baseline nozzle. From Table II it is clear that the 2tab configuration with $8 \%$ blockage area produced the biggest loss in performance. This nozzle configuration also produced the strongest induced counter-rotating vorticity. The nozzle configuration with 6-tabs produced the lowest performance loss and at the same time produced the strongest entrained mass flux in the potential core region of jet flow. All other tab configurations produced similar levels of performance loss.

\section{Conclusions}

This paper has examined the process of enhanced mixing in an unheated supersonic jet through the introduction of counter-rotating streamwise vorticity. The vorticity was introduced experimentally by placement of prism shaped devices (i.e. tabs) on the trailing edge of a model axisymmetric nozzle. Numerical simulations with a Navier-Stokes code without turbulence model were performed to separate out the influence between normal turbulent shear layer mixing processes from those produced by induced streamwise counterrotating vorticity introduced into the nozzle flow at regions comparable to the experiment.

Nozzles were designed that had 2, 4, and 6 tabs located on the nozzle lip. The design was such that induced vortex strength was diminished with increasing tab count, but total vorticity was held constant. The experiments unfortunately did not measure initial flow cross-stream velocity to validate this statement. The numerical simulations were performed with varying degrees of induced vortex strength to study the effect on mass flow entrainment. Experiments were also conducted with 2-tabs with different levels of induced vorticity.

The results show a striking similarity in the flow evolution between numerical and experimental jets, particularly in the jet potential core region. Even the mass flow entrainment rates are similar in this region of flow. Because of complications associated with computational boundaries, the comparison between experiment and simulation degrades by 8 jet diameters. The results of this study show similar values for mass entrainment relative to previous investigations

The nozzle with 6-tabs produced the least performance loss, while achieving the largest growth rate in the initial region of jet development. The nozzles with only two tabs produced the largest levels of mass flow entrainment relative to the 
baseline nozzle, but this occurs beyond the jet potential core. The difference in behavior between 2 and 6 tabs has strong implications on expected acoustic suppression characteristics. The2-tab nozzles would be expected to produce the largest reduction in noise around the baseline nozzle's spectrum peak amplitude level. However, the 6-tab nozzle could very well provide the best overall performance in the EPNL metric, where it is important to reduce mid-band frequencies.

Future experimental and numerical studies are required to more clearly align the initial induced vorticity field. Future experiments will use PIV imaging to measure the cross-stream vectors. Acoustic and performance measurements in the JNL LSAWT Low Speed Aeroacoustic Wind Tunnel) will be performed on larger nozzles with high temperature flow in simulated forward flight. Also the numerical simulations will be performed with more extensive computational boundaries to avoid their obvious interference noted in the present study. Also an attempt will be made to include a turbulence model in the numerical simulations.

The present study provides strong encouragement to utilize numerical simulations for this type of research. Based on the results it appears promising that a semi-empirical model could be developed relating tab design to mixing enhancement and jet noise.

Acknowledgement: C.E. Grosch was supported by the National Aeronautics and Space Administration under NASA Contract No. NAS1-97046, Task Order 302 while in residence at the Institute for Computer Applications in Science and Engineering (ICASE), NASA Langley Research Center, Hampton, VA 23681-0001.

\section{References}

1. Dolling, D.S., Foumier, E., and Shau, Y.R.. "Effects of Vortex Generators on the Growth Rate of a Compressible Turbulent Shear Layer", AIAA Paper No. 90-1979, 1990.

2. Drummond, J.P., Carpenter, M.H., Riggins, D.W., and Adams, M.S., "Mixing Enhancement in a Supersonic Combustor", AIAA Paper No. 89-2794, 1989.

3. Ahuja, K.K., and Brown, W.H., "Shear Flow Control by Mechanical Tabs", AIAA Paper No. 89-0994, 1989.

4. Samimy, M., Zaman, K.B.M.Q., and Reeder, M.F., "Effect of Tabs On The
Flow and Noise Field of An Axisymmeric Jet", AIAA J, 31, 609 (1993).

5. Zaman, K.B.M.Q., Reeder, M.F., and Samimy, M., "Control of an Axisymmetric Jet Using Vortex Generators", Phys. Fluids, 6, 778, 1994.

6. Seiner, J.M., "Fluid Dynamics and Noise Emission Associated With Supersonic Jets", Studies In Turbulence, Springer Verlag, pp 297-323, 1991

7. Peyret, R. and Taylor, T.D., "Computational Methods for Fluid Flow", Springer-Verlag, New York Heidelberg Berlin, 1983.

8. Grinstein, F.F., Gutmark, E.J., Parr, T.P., Hanson-Parr, D.M., and Obeysekare, U., "Streamwise and Spanwise Vortex Interaction in an Axisymmetric Jet. A Computational and Experimental Study", Physics of Fluids, 8, 1515 (1996).

9. Grosch, C.E., Seiner, J.M., Hussani, M.Y., and Jackson, T.L.,"Numerical Simulation of Mixing Enhancement in a Hot Supersonic Jet", Phys. Fluids, 9, 1125, 1997.

Table I. Maximum value of the magnitude of the cross-plane velocity vector as a function of downstream distance for various cases.

\begin{tabular}{|ccccc|}
\hline Z/D & 2-Tabs & 4a-Tabs & 4b-Tabs & 4c-Tabs \\
\hline 1 & 46.06 & 47.43 & 35.69 & 45.62 \\
3 & 20.56 & 29.58 & 23.21 & 35.18 \\
5 & 15.94 & 22.80 & 17.12 & 38.31 \\
8 & 16.32 & 50.98 & 39.32 & 50.85 \\
\hline
\end{tabular}

Table II. Nozzle Discharge Coefficient Summary

\begin{tabular}{|c|c|c|}
\hline No. of Tabs & $\begin{array}{c}\text { \% Area } \\
\text { Blockage }\end{array}$ & $\begin{array}{c}\text { Cd, Relative to } \\
\text { Baseline }\end{array}$ \\
\hline 2 - Fence & 3.57 & 0.932 \\
\hline 2 & 3.00 & 0.938 \\
\hline 2 & 8.00 & 0.901 \\
\hline 4 & 3.00 & 0.942 \\
\hline 6 & 3.00 & 0.953 \\
\hline
\end{tabular}




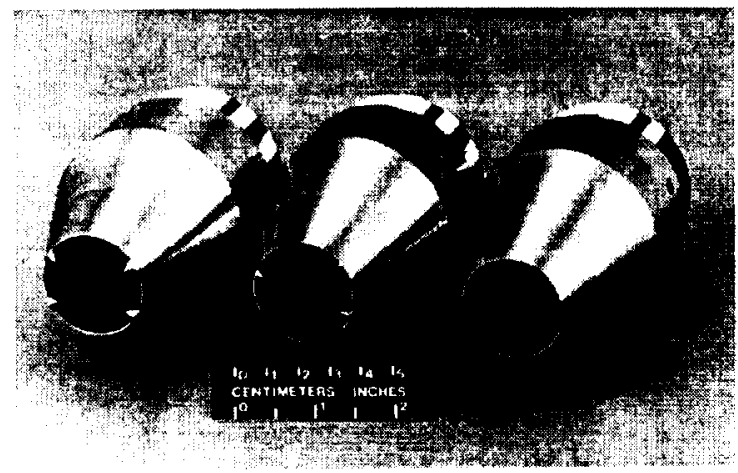

Figure 1. Photograph of baseline nozzle and nozzles with 4 and 6 tabs with $3 \%$ area blockage.

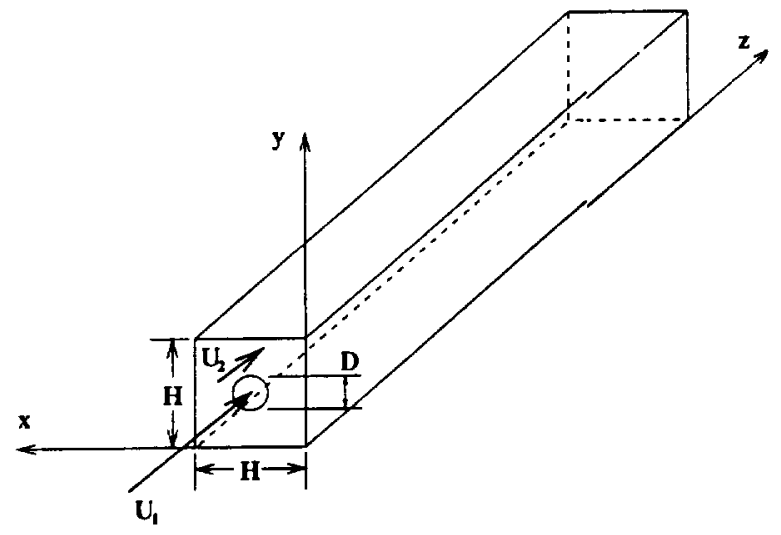

Figure 2. Schematic of computational channel and coordinate system.

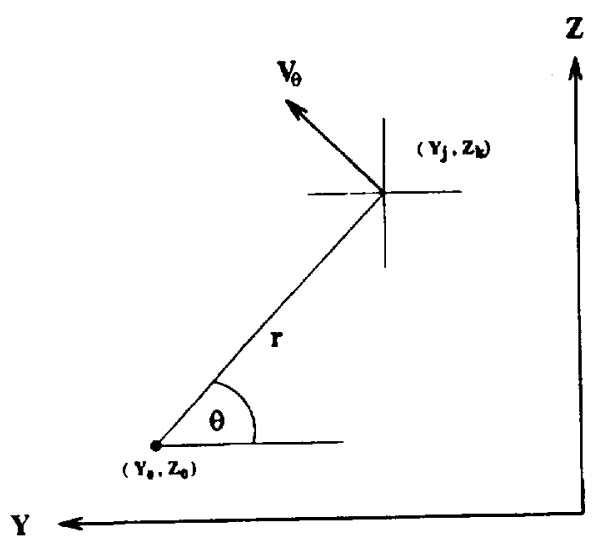

Figure 3. Sketch illustrating basic geometry of model vortices.

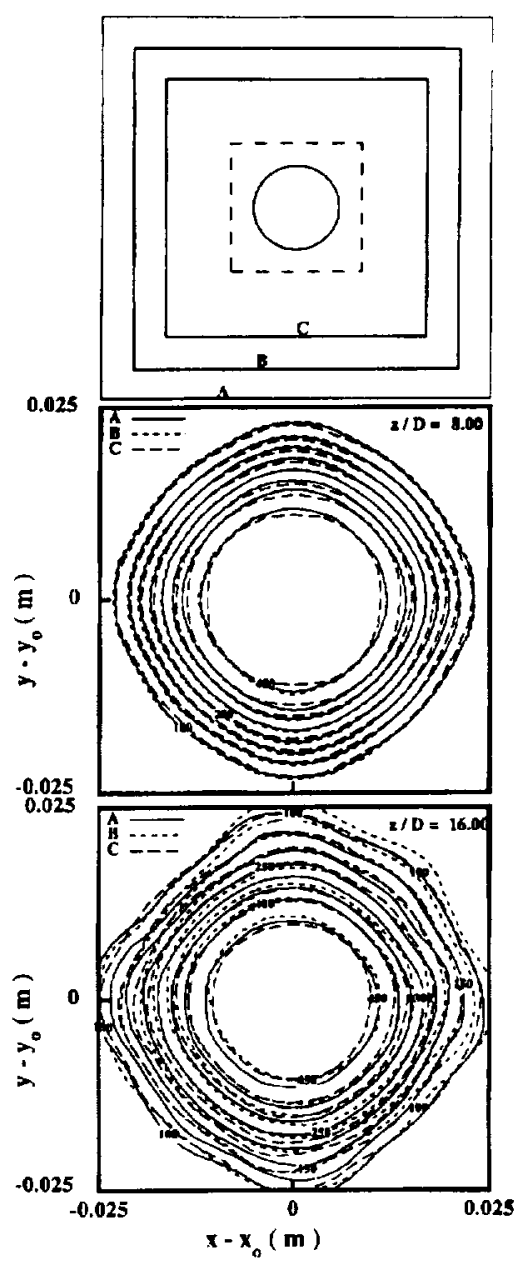

Figure 4. Results of the computational boundary sensitivity study.

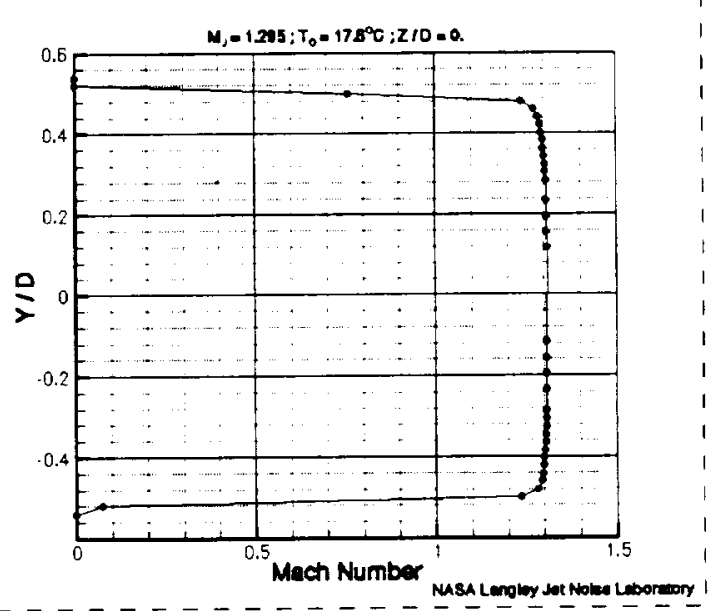

Figure 5. Jet exit Mach number profile of baseline nozzle. 


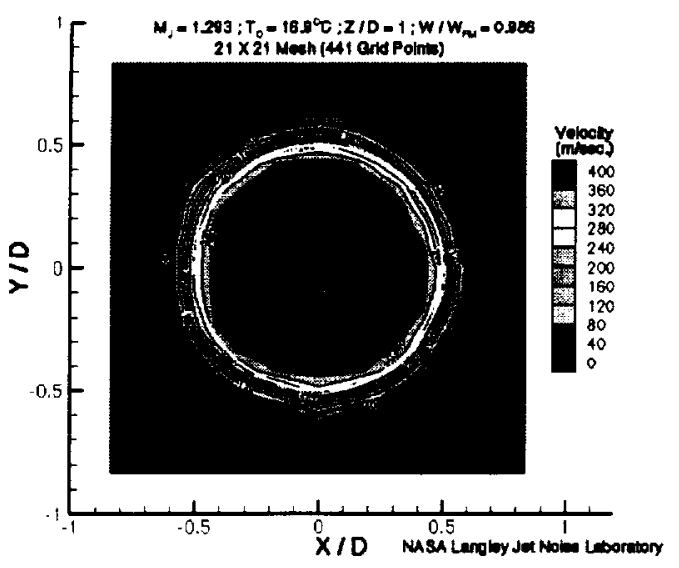

a. $z / D=1$

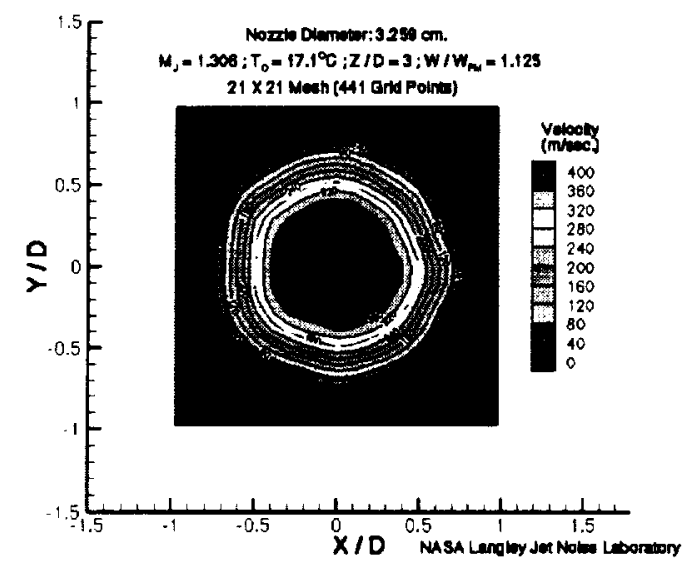

b. $z / D=3$.

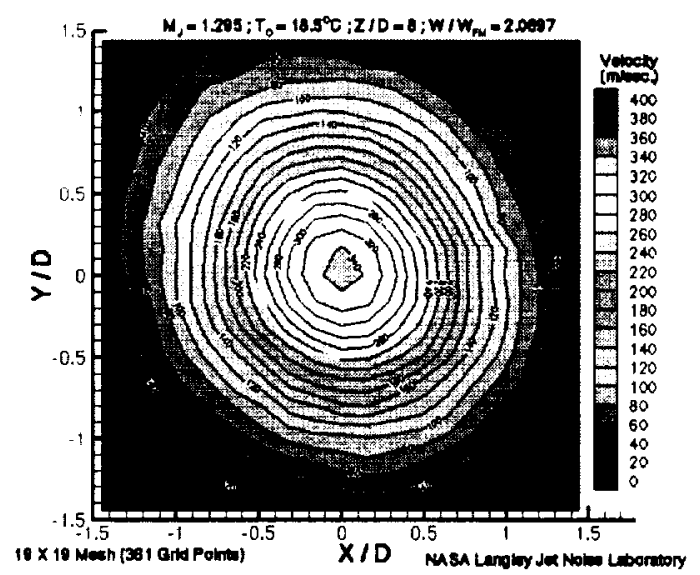

c. $z / D=8$.

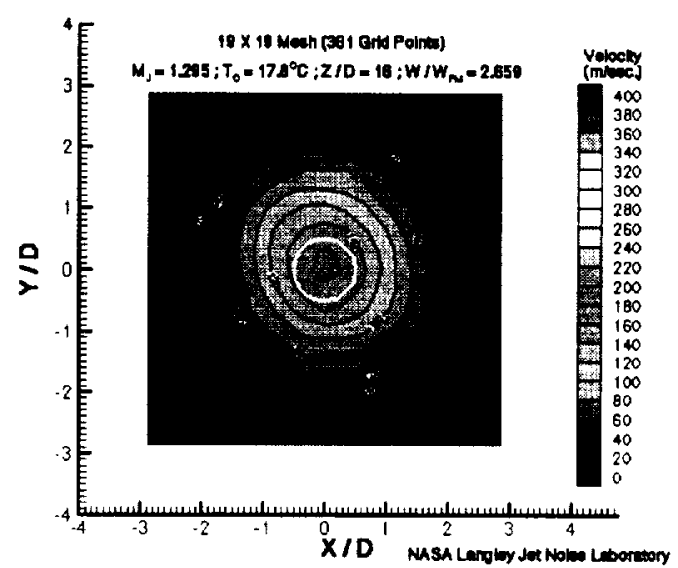

d. $z^{\prime} D=16$.

Figure 6. Baseline nozzle axial velocity contours.

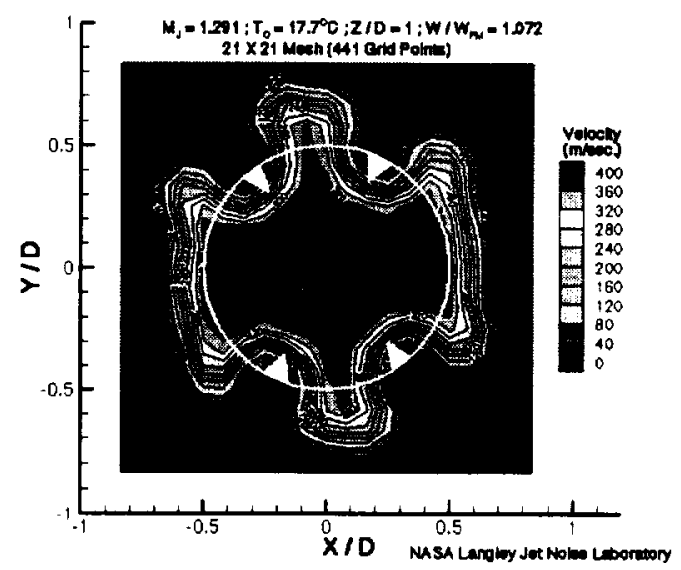

a. $\mathrm{z} / \mathrm{D}=1$.

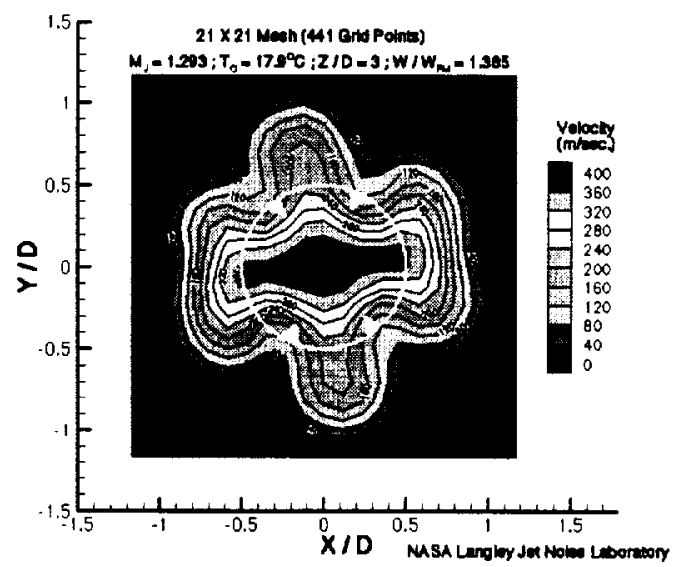

b. $z / D=3$. 


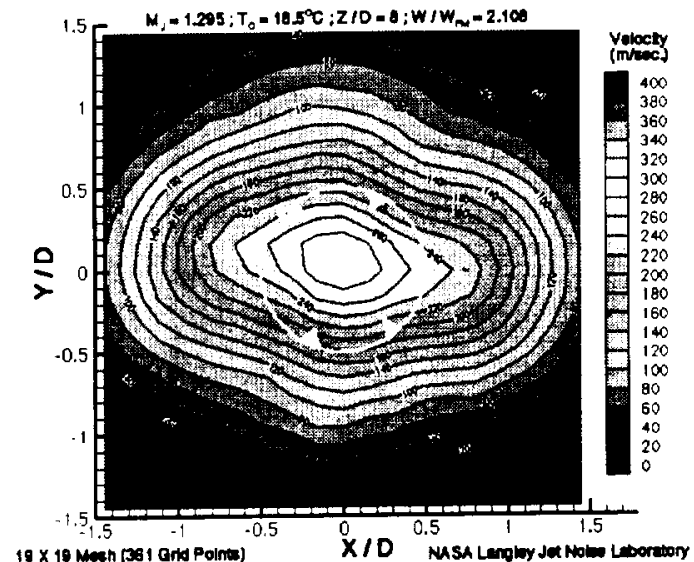

c. $z / D=8$.

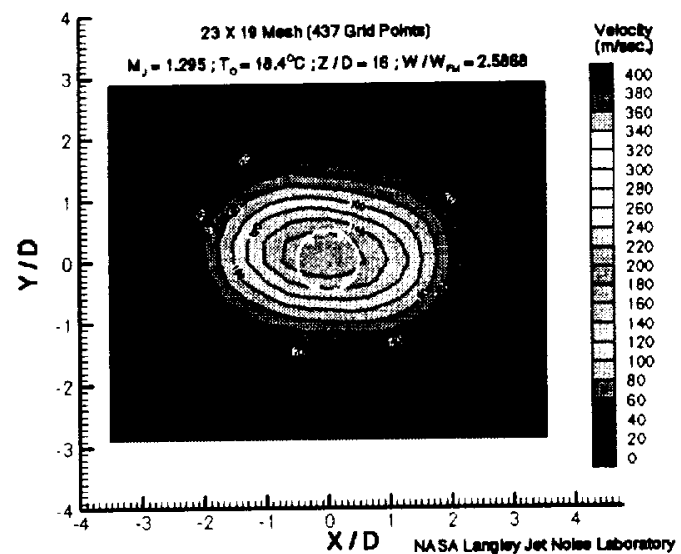

d. $z / D=16$

Figure 7. Axial velocity contours of nozzle with 4tabs with $3 \%$ blockage area.

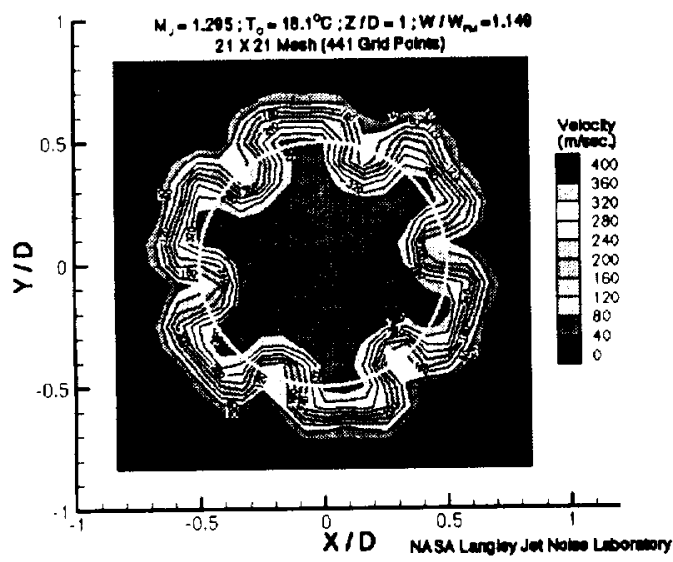

a. $z / D=1$

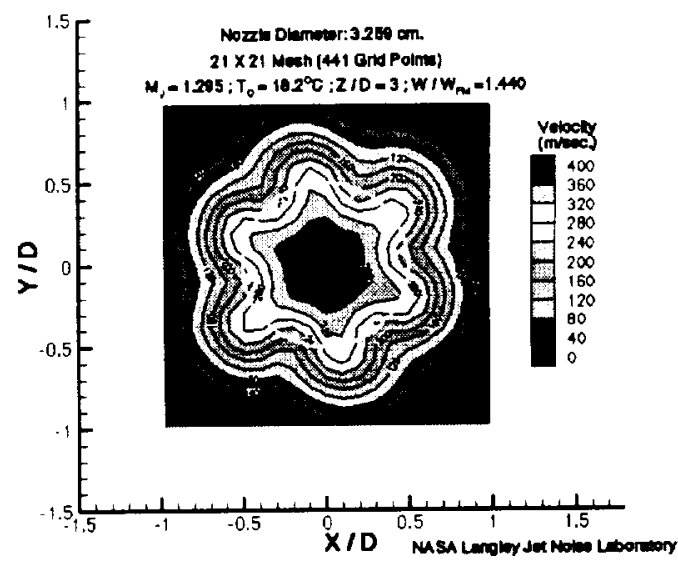

b. $z / D=3$

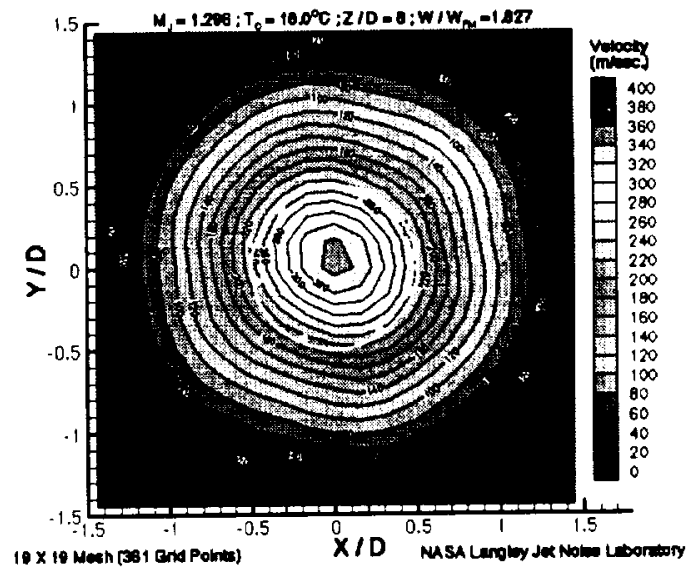

c. $z / D=8$.

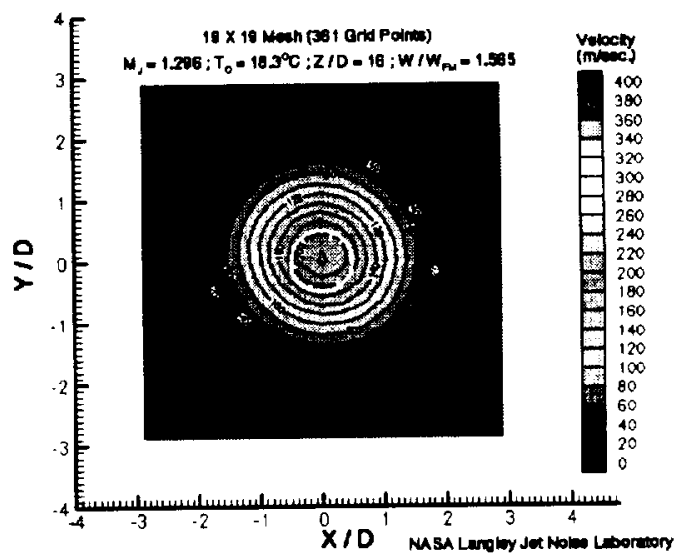

d. $z / D=16$.

Figure 8. . Axial velocity contours of nozzle with 6tabs with $3 \%$ blockage area. 


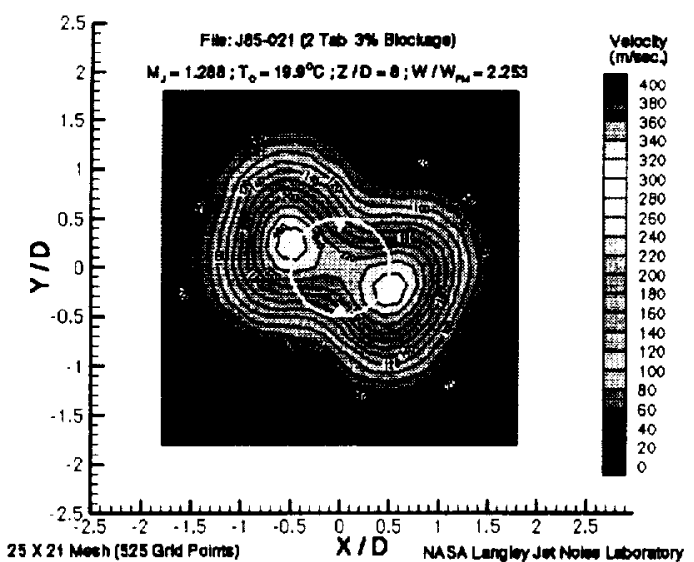

a. $z / D=8$

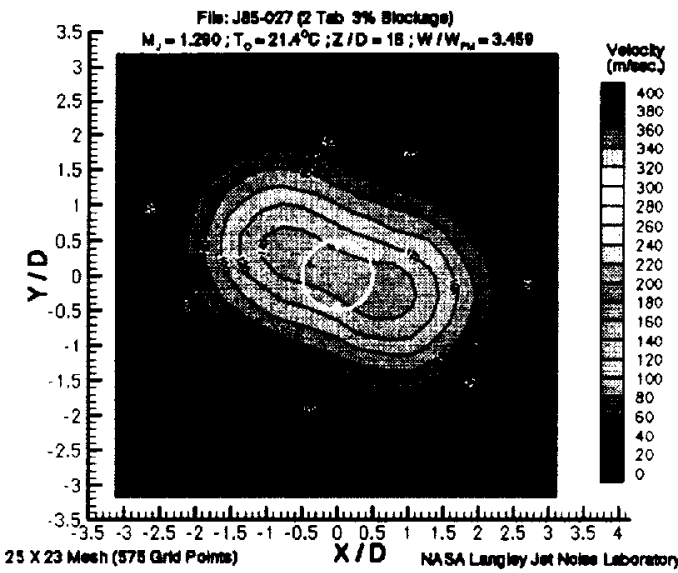

b. $z / D=16$.

Figure 9. . Axial velocity contours of nozzle with 2tabs with $3 \%$ blockage area.

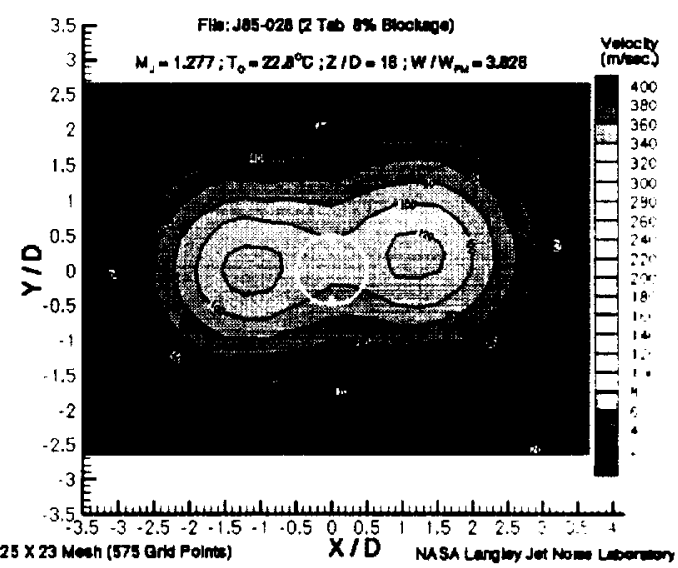

a. $z / D=8$.

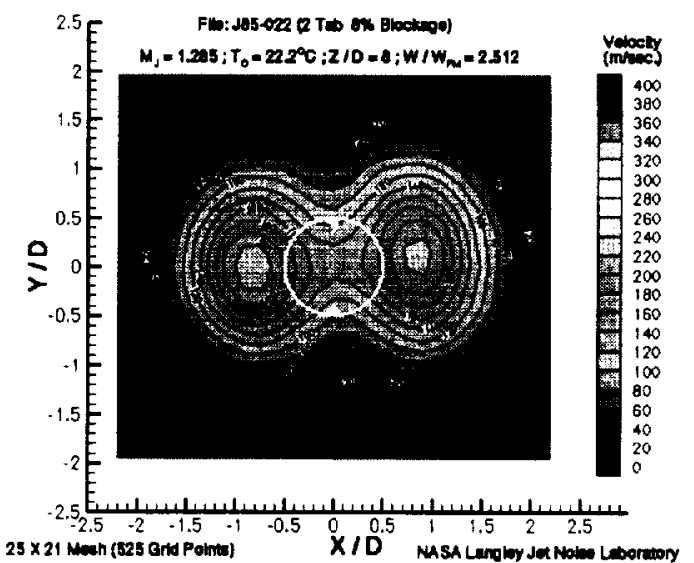

b. $z / D=16$.

Figure 10 . Axial velocity contours of nozzle with 2 -tabs with $8 \%$ blockage area.

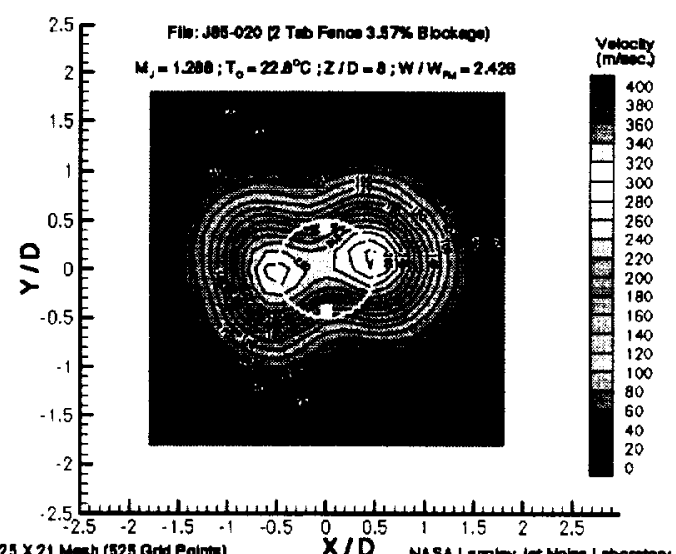

a. $z / D=8$.

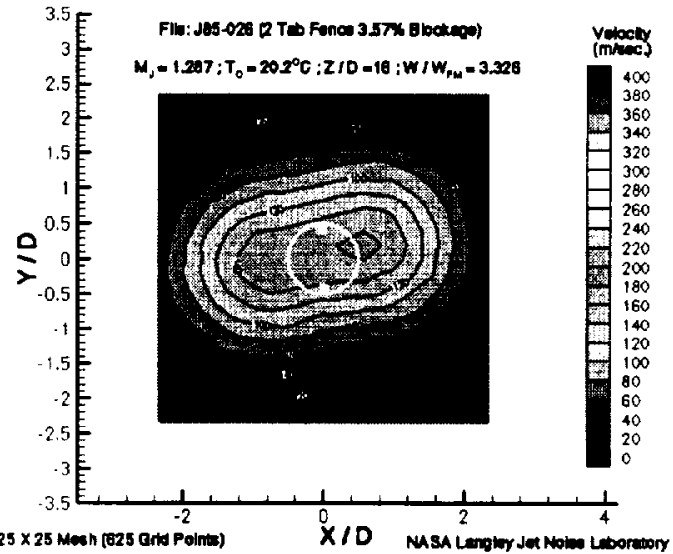

b. $z / D=16$.

Figure 11. Axial velocity contours of nozzle with 2-fence tabs with $3.57 \%$ blockage area. 

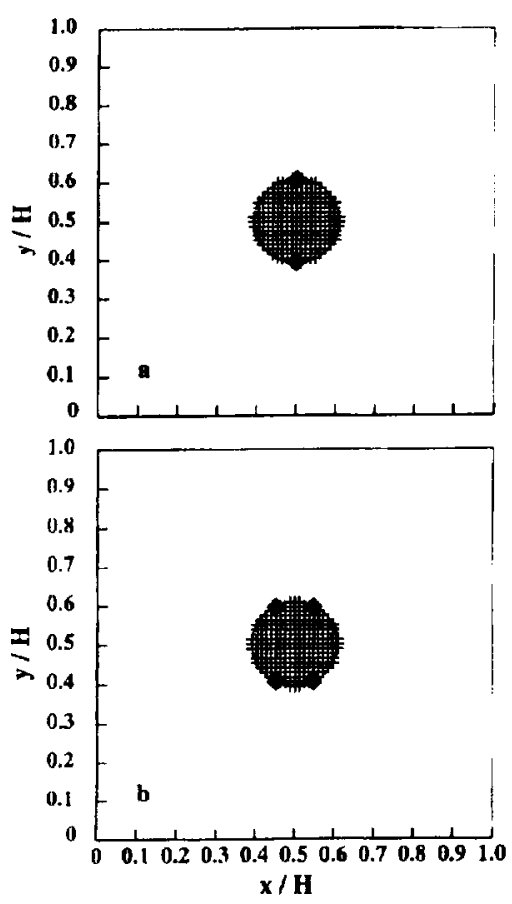

Figure 12. Tab placements in computational model.

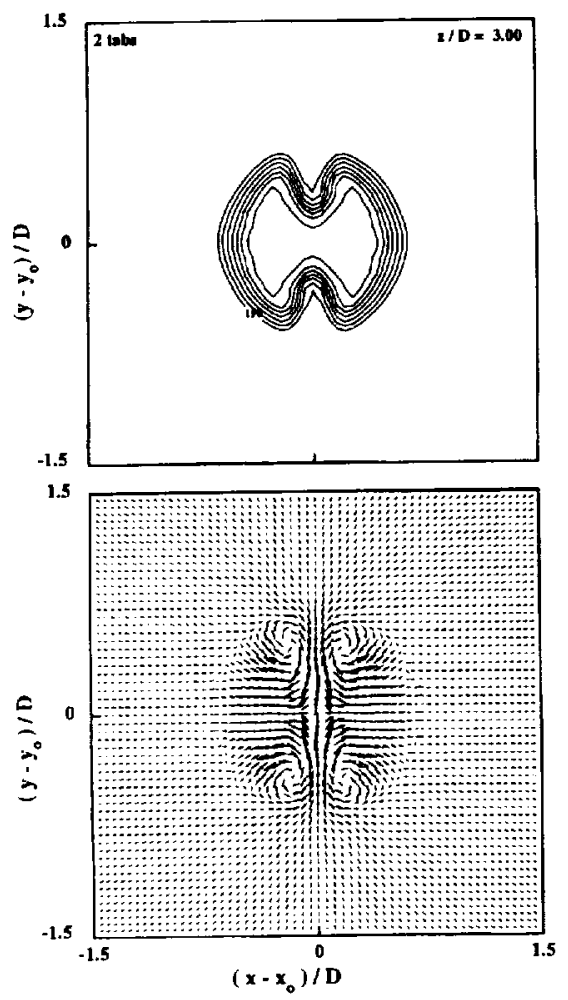

Figure 13. $(u, v, w)$ for 2-tabs at $z / D=3$.

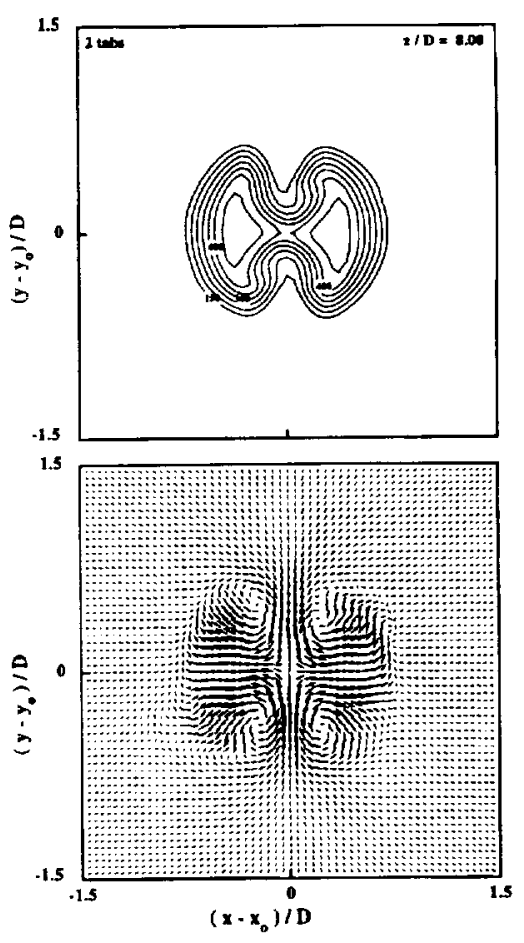

Figure 14. $(u, v, w)$ for 2-tabs at $z / D=8$.

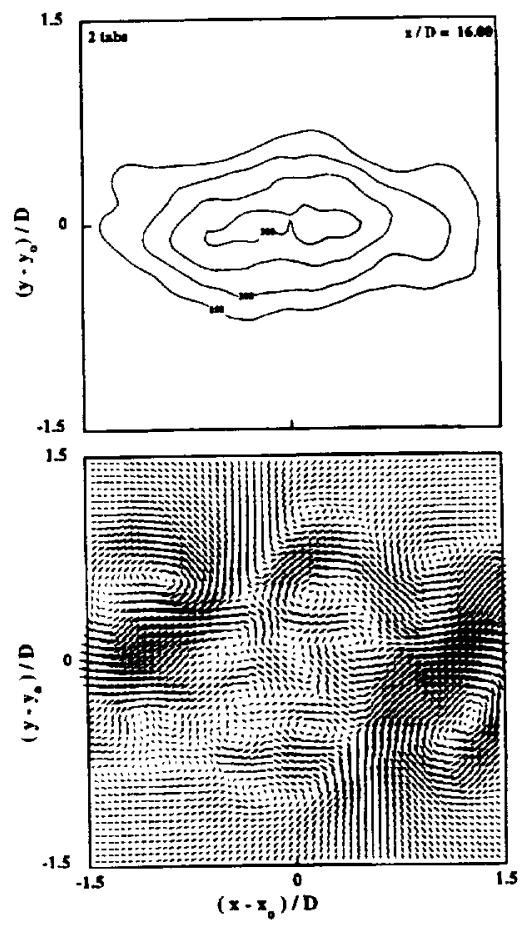

Figure 15. $(u, v, w)$ for 2-tabs at $z / D=16$. 


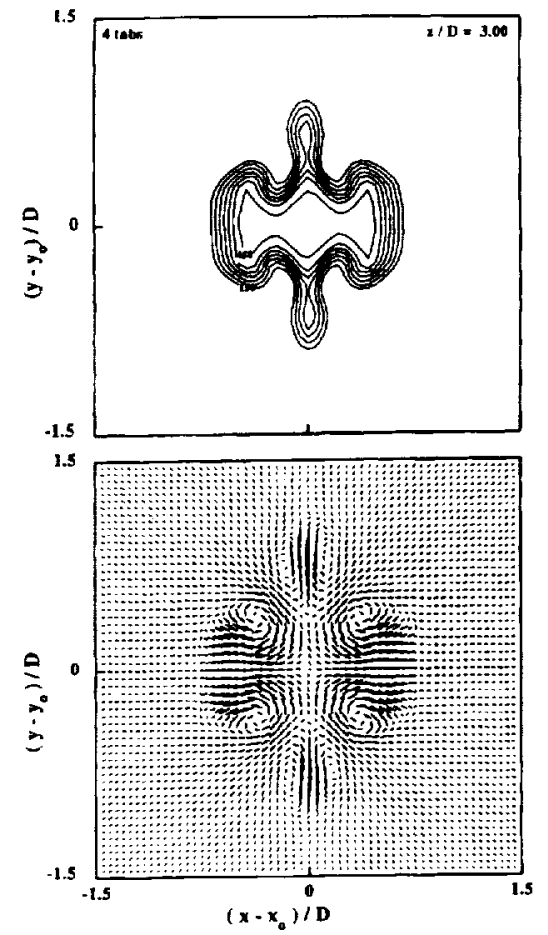

Figure 16. $(u, v, w)$ for 4 -tabs at $z / D=3$.

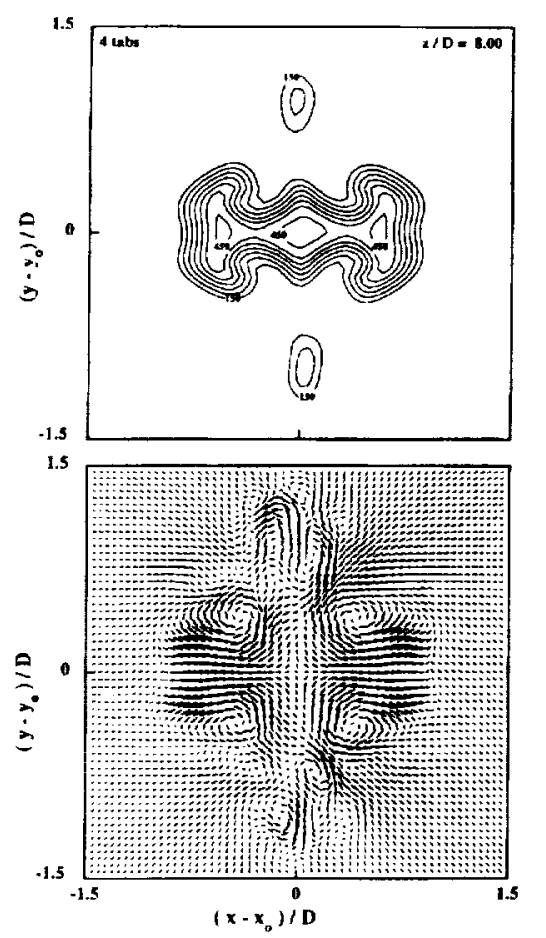

Figure 17. $(u, v, w)$ for 4 -tabs at $z / D=8$.

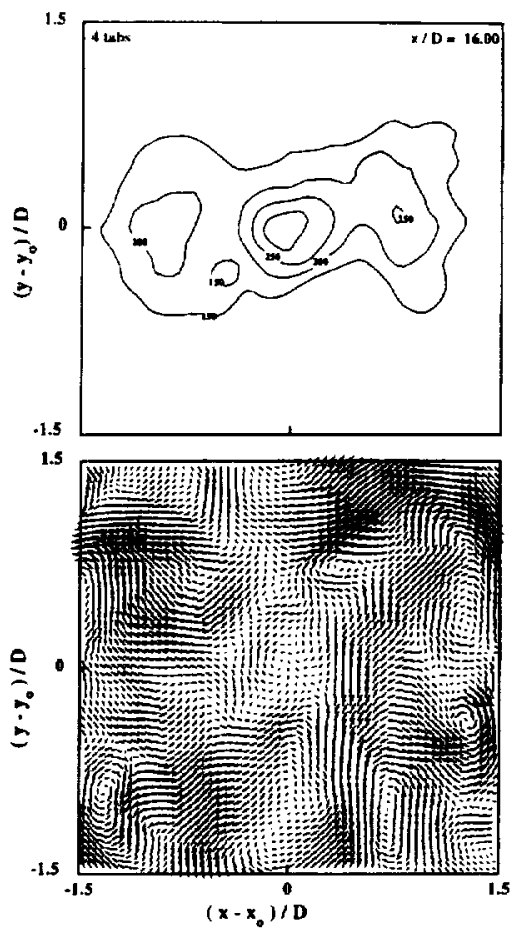

Figure 18. $(u, v, w)$ for 4-tabs at $z / D=16$.

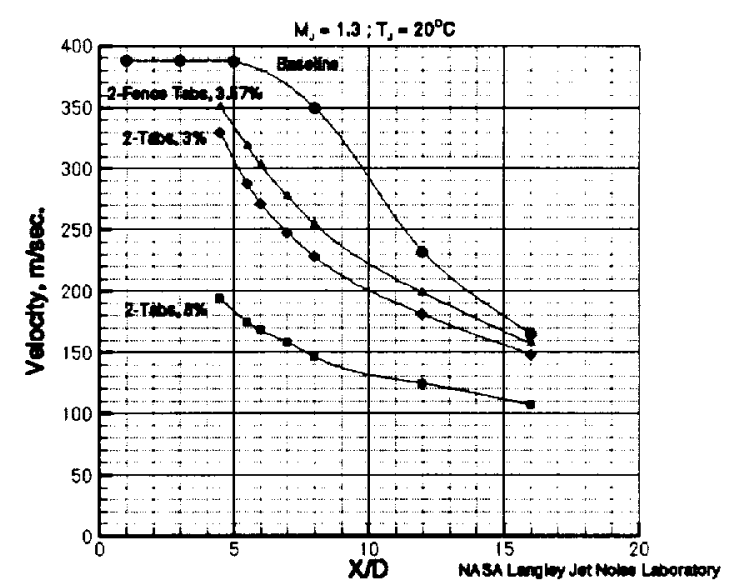

Figure 19. Centerline axial velocity profiles of baseline nozzle and those with 2-tabs. 


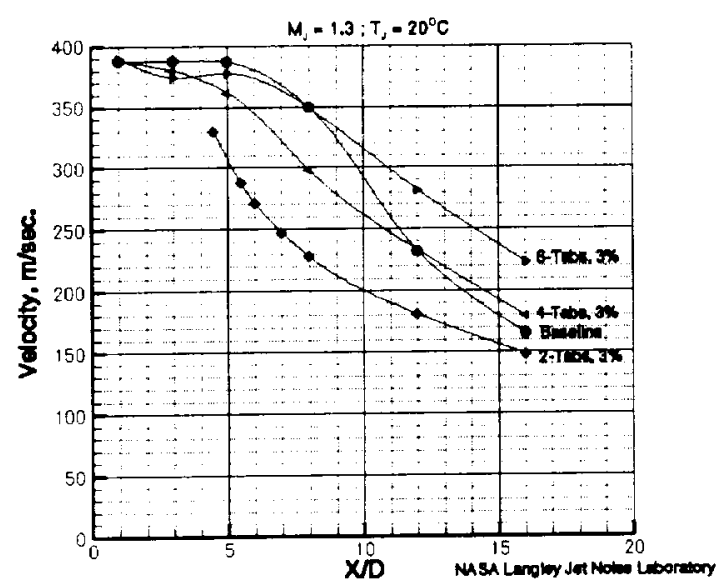

Figure 20. Centerline axial velocity profiles of baseline nozzle and those with 2,4 , and 6tabs with $3 \%$ blockage area.

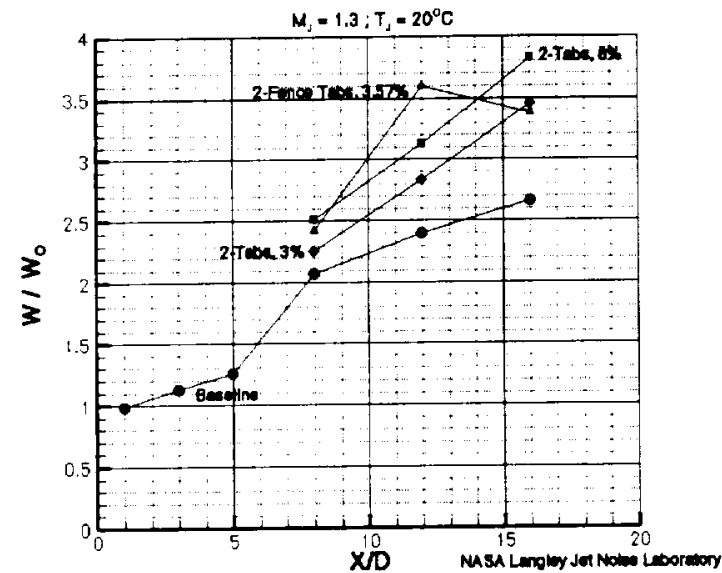

a. baseline and 2-tab nozzles

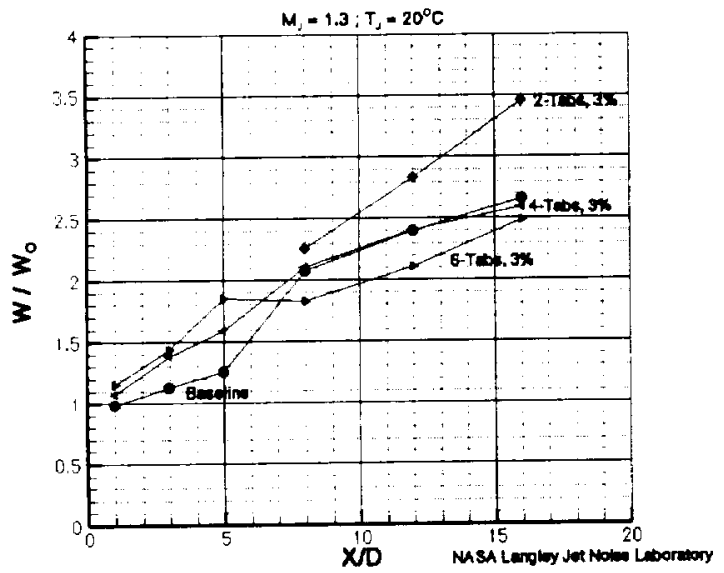

b. baseline and 2, 4, and 6-tab nozzles

Figure 21. Measured mass flow entrainment normalized by jet exit mass flow rate.

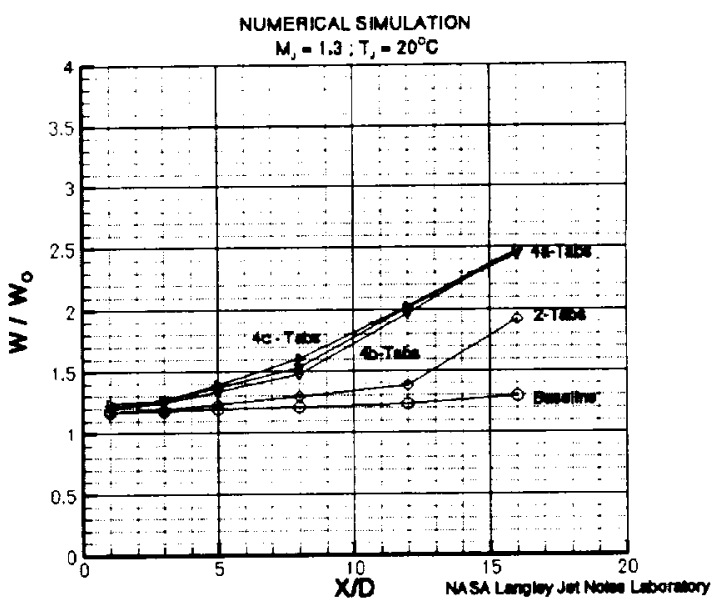

Figure 22. Computed mass flow entrainment normalized by jet exit mass flow rate.

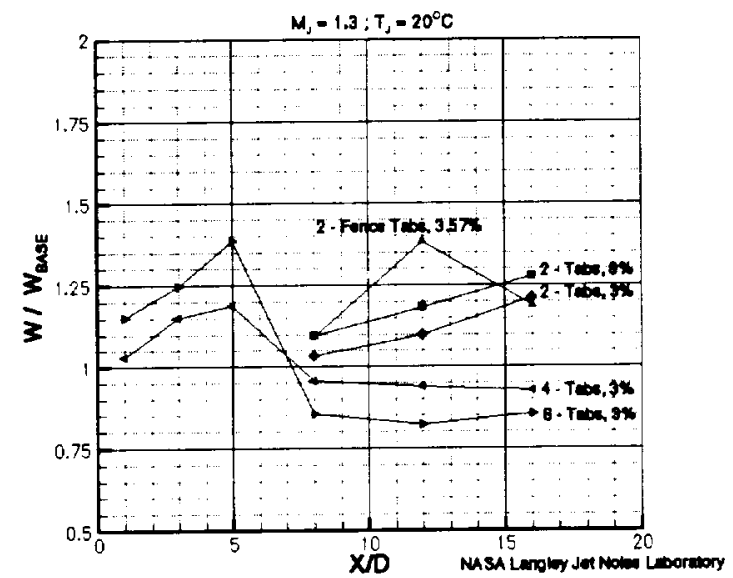

Figure 23. Measured mass flow entrainment normalized by baseline nozzle mass flow rate at same axila station.

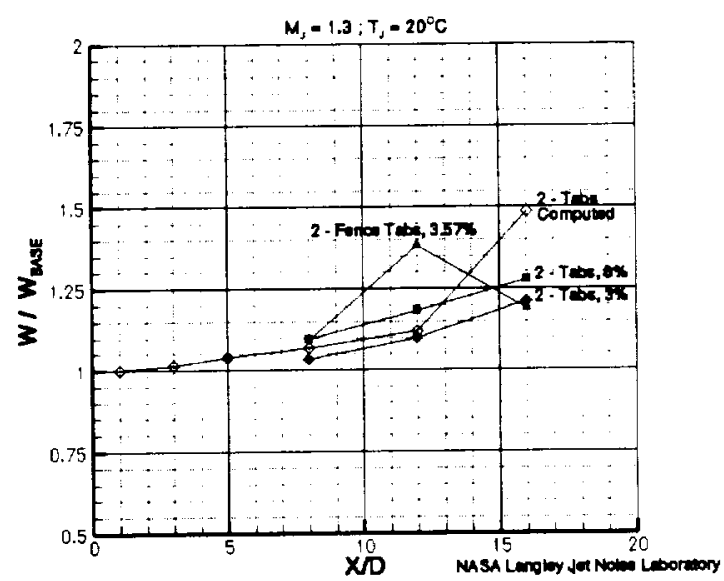

a. 2-tab nozzle configurations 


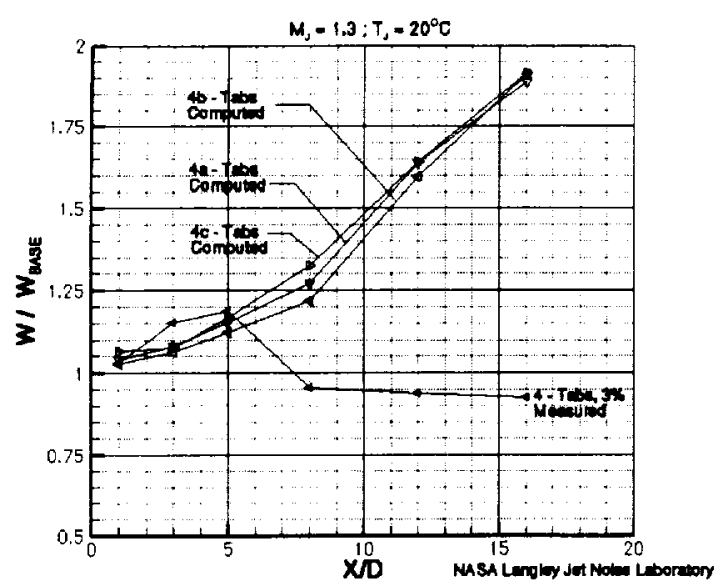

b. 2 and 4-tab nozzle configurations

Figure 24. Numerical and experimental comparison of mass flow entrainment rates normalized by baseline nozzle at same axial station. 